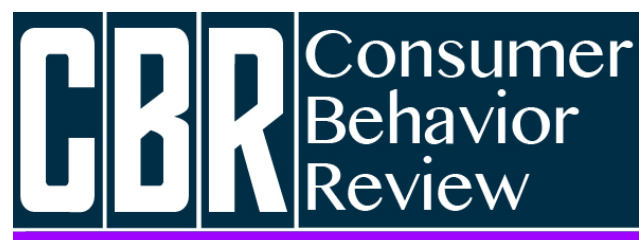

Revista Comportamento do Consumidor
Nascimento, T. M. (2019). Examinando o domínio do comportamento pró-ambiental na promoção do bemestar individual e coletivo. Consumer Behavior Review, $3(1), 27-41$.
ISSN: 2526-7884

Editor: Prof. Dr. Marconi Freitas da Costa

Email da revista: cbr@ufpe.br
Avaliação: Double blind review

Recebido: 23 de setembro de 2018

Aceito: 29 de março de 2019

\title{
EXAMINANDO O DOMÍNIO DO COMPORTAMENTO PRÓ-AMBIENTAL NA PROMOÇÃO DO BEM-ESTAR INDIVIDUAL E COLETIVO
}

Thays Martins do Nascimento

Thays Martins do Nascimento é Mestre em Administração e Negócios com ênfase em Marketing pela Pontifícia Universidade Católica do Rio Grande do Sul. E-mail: thaysdn@gmail.com. A autora agradece aos avaliadores pelos comentários para melhoria do artigo.

\begin{abstract}
Resumo
A análise do comportamento do consumidor está no centro de uma evolução sobre o comportamento ambiental e sustentável da sociedade. Os comportamentos próambientais ajudam a minimizar o impacto negativo do homem ao meio ambiente. Esta pesquisa delineia uma estrutura conceitual que se baseia nas normas sociais, valores e atitudes pró-ambientais para promover um comportamento pró-ambiental com contribuições para a pesquisa transformativa do consumidor de bem-estar pessoal e coletivo. Além disso, para entender melhor como se pode estimular esse comportamento em várias atividades de marketing, são discutidos como as empresas e sociedade podem se beneficiar desses comportamentos pró-ambientais, permitindo que as organizações explorem melhor suas vantagens.

Palavras-chave: Bem-estar, Comportamento pró-ambiental, Atitudes pró-ambientais, Pesquisa Transformativa do Consumidor.
\end{abstract}

Esta obra está licenciada com uma Licença Creative Commons Atribuição 4.0 Internacional.

\section{INTRODUÇÃO}

Devido ao aumento de forma constante ao longo da última década do impacto negativo das ações humanas no meio ambiente (IPCC, 2014) o tema do desenvolvimento sustentável se tornou global e preocupante. Tornando essencial se compreender as dinâmicas sociais que podem encorajar a mudança de estilos de 
vida (Steentjes, Kurz, Barreto, \& Morton, 2017) para hábitos mais sustentáveis. Para incentivar o consumidor a adotar esse estilo de vida, entender como a influência social opera, tendo em vista que ela atua claramente como um motivador de intervenções para promover o comportamento pró-ambiental (CPA) é importante para a disseminação desse comportamento.

Em seu trabalho, Abrahamse e Steg (2013) realizaram uma meta-análise que compara a eficácia das abordagens de influência social para incentivar ações de conservação de recursos, seus resultados apontam que a influência social foi efetiva no comportamento ambiental. Ainda identificam seis abordagens que são frequentemente usadas: (i) uso de normas sociais; (ii) liderança de blocos e redes sociais; (iii) elaboração de compromissos públicos; (iv) modelagem; (v) o uso da comparação social na provisão de feedback; e (vi) provisão de feedback sobre o desempenho do grupo (Abrahamse e Steg 2013) tendo em vista o foco deste trabalho, a influência social em relação ao CPA, abordaremos como as normas sociais podem ou não ajudar na disseminação desse comportamento.

Em específico as normas sociais são definidas como "regras e padrões que são entendidos por membros de um grupo e que orientam e/ou limitam o comportamento humano" (Cialdini e Trost, 1998, p. 152). Tendo em vista que a literatura sobre CPA fornece informações importantes sobre como as ações de comportamento contribuem para uma conduta ambientalmente amigável na sociedade (Barbarossa, De Pelsmacker, \& Moons, 2017; Ertz, Karakas, \& Sarigöllü, 2016; Kumar, Manrai, \& Manrai, 2017). Ainda se questiona sobre o que molda a predisposição de um indivíduo para adotar um CPA. Portanto analisar possíveis antecedentes do CPA se torna crucial para uma sociedade em mudança, em busca de um futuro mais sustentável (Coelho, Pereira, Cruz, Simões, \& Barata, 2017).

Em alguns estudos já foi constatado que comportamentos pró-ambientais podem ser influenciados por outras pessoas e pela personalidade do próprio consumidor (Barbarossa et al., 2017; Binder \& Blankenberg, 2017; Johe \& Bhullar, 2016). Em Leary, Vann e Mittelstaedt (2017) se avaliou que consumidores de alta influência percebida não só são mais propensos a praticar o comportamento pró-ambiental, mas também a participar de causas ambientais e encorajar os outros a fazerem o mesmo.

Um importante fator que pode facilitar comportamentos pró-ambientais é o conceito de valores biosféricos, que é definido como uma orientação de valor em que as pessoas julgam os fenômenos com base em custos ou benefícios para os ecossistemas ou a biosfera (Martin e Czellar, 2017). Os valores biosféricos fazem parte da orientação do valor do Universalismo, caracterizando-se por uma preocupação pelo bem-estar da sociedade e do mundo em grande escala e pela natureza (Schultz \& Zelezny, 1998; Schwartz, 1992; Schwartz et al., 2012). Ainda é preciso compreender se o consumidor se sente bem após ter esse comportamento positivo, ou se ele busca esse tipo de realização de bemestar social (Schmitt, Aknin, Axsen, \& Shwom, 2018), pois manter um consumo consciente pode trazer bem-estar ao consumidor (Kaida \& Kaida, 2016; Welsch \& Kühling, 2018). A questão de como melhorar o bem-estar social e alcançar o desenvolvimento industrial é um desafio fundamental para a sustentabilidade, e a busca da resolução desses conflitos resultará em melhores resultados de saúde e desenvolvimento ambiental, social e economicamente sustentável (Yang et al., 2014).

Embora uma intervenção de norma social possa ser efetiva na mudança de comportamentos para melhorar os resultados ambientais, a maioria das métricas avaliadoras não medem os efeitos de conformidade ou desvio no bem-estar subjetivo (Farrow, Grolleau e Ibanez 2017). Assim, mesmo que uma intervenção da norma social seja efetiva na mudança de comportamento em uma direção desejável, isso poderia chegar a um custo psicológico que mitigue outras melhorias no bem-estar (Farrow et al., 2017; Kjell, 2011); é preciso identificar o custo dos comportamentos ao bem-estar e avaliar seu impacto positivo ou negativo ao consumidor.

Essas pesquisas demonstram a relevância da influência de fatores pessoais e externos no comportamento ambiental dos consumidores, assim como para o bem-estar social e individual dos consumidores. Porém, ainda é preciso estudar o papel de fatores afetivos que poderiam determinar a intenção de se engajar no comportamento pró-ambiental, e até que 
ponto isso pode ser parcialmente refletido em medidas de auto identidade (Carfora et al., 2017). Os valores são também analisados por Groot e Steeg (2010) onde indicam que o papel dos valores é visto também como fatores motivacionais ao CPA.

Muitas organizações governamentais e não governamentais projetam e implementam campanhas para promover o comportamento pró-ambiental (Broek, Willem e Steg 2017). À luz desse desafio, é oportuno compreender melhor os fatores que contribuem para práticas sustentáveis de consumo, para ajudar na promoção e incentivo de CPAs. Através deste estudo se busca formular um framework teórico com proposições de pesquisa, de ações que levam o consumidor efetivamente a um comportamento pró-ambiental. Fornecer aos gestores sugestões mais práticas de ações, identificar algumas variáveis que não só tenham um impacto no comportamento ambiental, mas também possam permitir que as organizações exerçam influência em seus esforços de marketing (Huang, 2016), agregando valor a sua marca/serviço e em busca de vantagem competitiva.

Alguns estudos indicam a inclusão dessas variáveis ao contexto do comportamento próambiental, primeiro para ampliar o escopo de pesquisa e segundo pois, indicam que as normas sociais ajudam a explicar parte do comportamento pró-ambiental Lee et al. (2014). Em suas análises, (Abrahamse \& Steg, 2013), forneceram uma visão sobre os processos pelos quais as abordagens de influência social afetam o comportamento, e indicam a necessidade de uma pesquisa empírica mais sistemática que ligue os mecanismos de influência social à mudança de comportamento, e ainda se sugere que estudos futuros se beneficiariam com a inclusão de comportamentos objetivos, observando e registrando comportamentos reais de reciclagem.

Relevante considerar a forma como a visibilidade social poderia afetar os comportamentos pró-ambientais, já que um estudo recente mostrou que a força com que as identidades sociais predizem comportamentos pró-ambientais depende de serem visíveis para outros (Carfora et al., 2017). Ertz, Karakas e Sarigöllü, (2016) descrevem que há ainda muitas variáveis que valem a pena ser investigadas, como influências interpessoais (por exemplo, persuasão), publicidade, expectativas da comunidade e fatores legais e institucionais. Outros fatores também ressaltados são normas pessoais, crenças e valores, delineados na teoria da norma de crença de valor (Steg \& Vlek, 2009). Assim, embora seja claro que as normas sociais são um determinante confiável do comportamento próambiental, faz-se eco da chamada de outros autores para que mais estudos sejam feitos sobre como as normas devem ser melhor alavancadas (Miller \& Prentice, 2016).

Há de fato uma necessidade de entender como as normas sociais atuais se desempenham nas interações pessoais e as formas como esses processos podem (ou não) significar mudanças significativas nos estilos de vida (Steentjes et al., 2017). Devido a essas limitações de estudos atuais, este trabalho tem por objetivo analisar como as variáveis de normas sociais, atitudes e valores podem ser estudadas e aumentar o comportamento ambiental, buscando o bemestar do consumidor. Dado que uma única teoria de tomada de decisão não é susceptível de explicar bem o comportamento em todos os contextos, há uma necessidade especial de identificar os contextos que produzem regularidades comportamentais robustas e os elementos desses contextos que mais importam na determinação das capacidades preditivas dos modelos teóricos (Farrow et al., 2017).

Em resumo, o presente artigo tem como objetivo geral propor um framework teórico para a análise do comportamento próambiental e suas relações sociais, lançando luz nesta temática e contribuindo, portanto, para o desenvolvimento de novos esforços analíticos direta ou indiretamente relacionados a ela. Serão analisadas as normas sociais, os valores como sendo preditoras das atitudes e comportamentos pró-ambientais, e a relação do bem-estar do consumidor como uma consequência. 0 propósito é trazer o tema da sustentabilidade para discussão e responder o seguinte questionamento: Como as normas sociais e os valores afetam o comportamento e as atitudes ambientais, e é possível, para os consumidores, a partir dessas mudanças, esperar/obter bem-estar social ou individual?

Primeiramente, serão conceitualizadas as variáveis escolhidas para se analisar e, posteriormente, serão feitas algumas proposições de estudos futuros, buscando 
expandir as pesquisas sobre o comportamento pró-ambiental. A revisão de literatura deste artigo está organizada da seguinte forma: (1) comportamento pró-ambiental; (2) atitudes pró-ambientais; (3) normas sociais; e (4) bemestar. Na sequência, apresenta-se uma discussão sobre pesquisas, sugestões de estudos futuros e conclusão.

\section{MODELO CONCEITUAL E PROPOSIÇÕES DE PESQUISA}

\section{Comportamento Pró-Ambiental}

o comportamento pró-ambiental é considerado aquele que tem um impacto positivo na disponibilidade de materiais e energia também um comportamento que tem possui um menor impacto ou altera de maneira positiva a dinâmica dos ecossistemas ou da biosfera (Stern, 2000, p. 408). Definido também por ser um "comportamento que prejudique o ambiente o mínimo possível ou até beneficie o meio ambiente" (Steg \& Vlek, 2009b). A "ecologização" de comportamentos individuais é um componente importante na mitigação das mudanças climáticas e outros problemas ambientais causados pelo homem (Dietz et al., 2009; Schmitt et al., 2018).

Em seu estudo Stern, (2000), resume o CPA através de seu impacto e intenção, quando definido por seu impacto, o comportamento ambiental é um comportamento que pode mudar positivamente a disponibilidade de materiais, a energia ou a dinâmica dos ecossistemas, logo quando definido por sua intenção, o comportamento pró-ambiental é um comportamento realizado com o intuito de mudar positivamente o ambiente, do ponto de vista do ator.

A maioria dos estudos sobre comportamento pró-ambiental usa a definição orientada para o impacto como a variável dependente final. Além disso, essas pesquisas examinam um tipo de comportamento, particularmente o CPA pessoal dos indivíduos, como reciclagem, conservação de recursos ou consumo verde (Huang, 2016). Para (Johe \& Bhullar, 2016), prever um comportamento requer uma compreensão de como os indivíduos desenvolvem intenções, que são decisões conscientes, para envolver ou não em um comportamento específico. Essas intenções são antecedentes direto do comportamento (Ajzen, 1991a). Devem ser consideradas influenciadores de comportamentos pró-ambientais como valores e crenças, e assim trazendo benefícios ao consumidor como qualidade de vida e bemestar.

\section{Atitudes Pró-ambientais}

Rucker et al., (2014) propõem um conjunto de modelos que liga as atitudes ao comportamento, sugerindo que os indivíduos refletem suas atitudes para verificar se elas são guias por comportamento adequados. Há uma importância das atitudes, que afetam o comportamento dos consumidores no seu processamento de informações, e até a sua percepção básica. Como consequência, o estudo das atitudes e da persuasão tornou-se relevante nas pesquisas sobre comportamento do consumidor. Do ponto de vista da psicologia, as atitudes são disposições avaliativas dos indivíduos em relação a determinados objetos, questões ou pessoas (Schröder \& Wolf, 2017), que possuem aspectos cognitivos, afetivos e conativos, ou seja, crenças, sentimentos e tendências de ação, respectivamente, associadas a um objeto.

Sabe-se que a probabilidade de uma pessoa influenciar as atitudes de outra pessoa através da comunicação, seja através de mecanismos sistemáticos ou heurísticos, depende da similaridade inicial de suas atitudes. Se duas pessoas estiverem muito distantes em suas opiniões, as tentativas de influência, muitas vezes, serão infrutíferas ou podem até mesmo regredir (Schröder \& Wolf, 2017). Então, para uma pessoa que tenha atitudes pró-ambientais, será mais difícil influenciar alguém que nunca tenha pensado em ter uma atitude ambiental do que uma pessoa que simpatize com o tema.

Robinson \& Smith, (2002) descobriram que os consumidores conscientes relatavam mais atitudes, crenças e intenções de apoio para comprar produtos alimentares sustentáveis. Os autores propõem que as atitudes dos consumidores (avaliação geral da realização de um comportamento), o controle comportamental percebido (ou seja, a facilidade percebida de fazer um comportamento) e as normas (pressão social percebida de outros importantes e grupos de referência relevantes) vinculariam a identidade do consumidor (preditor) com as suas intenções (resultado) 
(Johe \& Bhullar, 2016). (Ertz et al., 2016) consideram as atitudes como crenças específicas do comportamento, essas atitudes refletem os tipos de predisposições subjacentes ao desejo de atuar com intenção pró-ambiental e que, portanto, podem influenciar a ocorrência de comportamento ambiental do consumidor, afetando diretamente o comportamento.

Porém, ainda há uma necessidade de esclarecimento conceitual de atitude (Wan et al., 2017). Por um lado, (Ajzen, 1991b) sugere que a atitude é a avaliação subjetiva de um comportamento individual e compreende dois componentes, ou seja, componentes experienciais (afetivos, sentimentos) e instrumentais (conhecimento, função). A atitude experiencial é o sentimento afetivo do indivíduo em relação ao comportamento (por exemplo, a reciclagem é um bom comportamento). A atitude instrumental se refere à avaliação de um indivíduo sobre os resultados do comportamento (por exemplo, a reciclagem pode reduzir a carga do aterro sanitário).

A atitude ambiental das pessoas parece, essencialmente, representar normas morais e altruístas. Ou seja, o interesse dos outros, o interesse próprio e outras considerações de utilidade parecem ser menos importantes para a conservação e, possivelmente, para outros comportamentos morais (Kaiser, 2006).

A atitude é conceitualizada como uma noção multidimensional e sua mensuração deveria incluir dimensões experienciais (sentimento, afetivo) e instrumental (benefícios, funções). Muitos estudos definiram a atitude em termos de dimensão experiencial para classificar o comportamento em questão de forma que seja considerado bom, sensível, agradável ou gratificante, entre outros. Esse conjunto de sentimentos afetivos é como uma dimensão hedônica da atitude do consumidor. Por exemplo, as autoridades públicas podem encorajar os cidadãos a realizar comportamentos de reciclagem enfatizando os sentimentos (isto é, bons, gratificantes, etc.) ou as consequências (isto é, economizando recursos, reduzindo a poluição, etc.) (Wan et al., 2017).

\section{Influência Social}

\section{Normas sociais e valores}

A influência social refere-se às formas em que o comportamento é afetado pelo que as outras pessoas fazem, ou pelo que as outras pessoas pensam. Diversas ideias e teorias de influência social foram aplicadas como parte de intervenções para incentivar a conservação de recursos, como o uso de normas sociais, aprendizagem social e processos de comparação social (Abrahamse \& Steg, 2013). As normas sociais são regras ou padrões de comportamento entre os membros de um grupo e podem ser categorizados de duas maneiras principais: como normas injuntivas (percepções sobre o que as pessoas devem fazer) e normas descritivas (o que as pessoas realmente fazem) (Bissing-Olson, Fielding, \& Iyer, 2016).

As normas sociais são concebidas como regras e padrões que são entendidos por membros de um grupo, e que orientam e/ou limitam o comportamento humano (Cialdini \& Trost, 1998). Podem distinguir-se dois tipos de normas sociais: as descritivas e as cautelares (Cialdini, Kallgren, \& Reno, 1991). As crenças sobre quais ações são comuns em uma determinada situação são definidas como normas sociais descritivas. As crenças sobre o que "deve" ser feito em uma determinada situação são referidas como normas sociais cautelares. Quando usado em intervenções para incentivar a conservação de recursos, as informações podem ser transmitidas sobre o que outras pessoas estão fazendo, e/ou o que outras pessoas aprovam ou desaprovam (Cialdini \& Goldstein, 2004).

Em alguns estudos, foi comparada a eficácia de diferentes abordagens de influência social para incentivar a conservação de recursos, através em uma meta-análise, descrevendo sobre os mecanismos que ligam a influência social à mudança de comportamento (Abrahamse \& Steg, 2013). Vários pesquisadores sugeriram que a eficácia das intervenções, para incentivar a conservação de recursos, poderia ser reforçada através da influência social, tais como normas sociais, aprendizagem social e comparação social (Cialdini, 2003).

As normas sociais são pistas que ajudam as pessoas a dar sentido às situações sociais, especialmente aquelas caracterizadas por um alto grau de ambiguidade, em termos de como as pessoas devem se comportar (Cialdini \& 
Goldstein, 2004). As pessoas aderem às normas sociais para obter aprovação social ou para evitar sanções sociais, de modo que esperam que outras pessoas gostem delas (Cialdini \& Goldstein, 2004; Keizer \& Schultz, 2018; Ruepert et al., 2016). Estudos mostram que o comportamento pode mesmo ser guiado por normas sociais quando o indivíduo não está ciente disso (Cialdini et al., 1991) , orientando o comportamento. Por exemplo, as pessoas são mais propensas a colocar lixo em ambientes desarrumados (ou seja, onde a norma descritiva em favor do lixo é saliente), mesmo quando a norma prevalecente favorece a falta de lixo.

As crenças ambientais representam as crenças gerais que as pessoas têm em relação ao ambiente natural. Em seus estudos (Huang, 2016; Lee, Kim, Kim, \& Choi, 2014) referem-se a crenças ambientais como preocupação, valores ou atitudes ambientais. Algumas medidas prevalentes de orientação pró-ambiental utilizam as crenças primitivas das pessoas sobre a natureza e o relacionamento da humanidade com ela (Dunlap, Van Liere, Mertig, \& Jones, 2000).

A motivação para se envolver em um comportamento pró-ambiental deve ser aumentada quando as pessoas percebem normas descritivas pró-ambientais mais positivas, em comparação com quando as pessoas percebem normas descritivas próambientais menos positivas. Em outras palavras, as normas do grupo podem ser, particularmente, importantes para o sentimento de orgulho sobre o comportamento ambiental para se traduzirem em ação pró-ambiental contínua (Bissing-Olson et al., 2016).

\section{Bem-estar}

Embora uma intervenção de norma social possa ser efetiva na mudança de comportamentos para melhorar os resultados ambientais, a maioria das métricas avaliadoras não medem os efeitos de conformidade ou desvio no bem-estar subjetivo (Farrow et al., 2017). Uma mudança cultural para a escolha de estilos de vida menos consumistas pode originar, não motivado por imperativos morais ou movimentos ambientais, mas pela busca do bem-estar humano (Brown \& Vergragt, 2016).

o Bem-estar é definido por ser o termo mais geral que abrange o quão bem os indivíduos estão fazendo na vida, incluindo dimensões sociais, de saúde, materiais e subjetivas de bemestar (Kitayama, Markus, \& Kurokawa, 2000; Searle, 2008). Assim, o bem-estar é um constructo amplo que reflete a avaliação global de uma pessoa sobre a qualidade de vida na sua perspectiva (Diener, Lucas, Schimmack, \& Helliwell, 2009). 0 bem-estar é considerado algo bom e desejável, e especificamente falando do bem-estar subjetivo (BES) ele considera a perspectiva da pessoa cuja a vida está sendo avaliada (Lucas, 2016).

É possível imaginar um cenário em que a compreensão central do bem-estar mudará através dos efeitos combinados da mudança de estilos de vida; da adaptação às realidades econômicas, tecnológicas e demográficas; e das novas práticas sociais emergentes (Brown \& Vergragt, 2016). Promover o bem-estar dos clientes pode ser especialmente importante entre os consumidores (Sheng, Siguaw, \& Simpson, 2016).

0 bem-estar do consumidor inclui sua satisfação com a vida, a qualidade de vida percebida e a felicidade geral (Davis \& Pechmann, 2013). Essa é uma medida que também pode ser usada nas pesquisas transformativas de serviços (Transformative Service Research - TSR), onde se defende a preocupação com o bem-estar dos consumidores e dos funcionários, e em como eles são afetados pelos serviços (Anderson et al., 2013).

Pesquisas extensas sobre o bem-estar sugerem que tal reestruturação pode, facilmente, incorporar uma mudança de estilos de vida consumistas. Em essência, uma transição além do consumismo implicaria uma evolução em toda a sociedade, em direção a diferentes estilos de vida e concepções de bemestar, bem como uma transformação do sistema. 0 discurso da sustentabilidade incorporou os conceitos de boa vida, bem-estar e felicidade como parte do questionamento do paradigma do crescimento econômico, como um caminho para o florescimento da sociedade (Brown \& Vergragt, 2016).

\section{Contribuição para a Pesquisa Transformativa do Consumidor}

Em 2005, um movimento pequeno, mas crescente, no campo da pesquisa do consumidor 
formou-se para deliberar questões-chave relacionadas ao bem-estar do consumidor. Este movimento, a pesquisa transformativa do consumidor (Transformative Consumer Research - TCR), encoraja os pesquisadores a enfrentar problemas sociais crônicos que afetam consumidores em todo o mundo (Bublitz et al., 2013).

Portanto, a TCR tem um futuro promissor como uma estrela em ascensão dentro do marketing, por causa de seu compromisso de melhorar de forma mensurável o bem-estar pessoal e social, bem como seu compromisso com o desenvolvimento da teoria do consumidor (Davis, Ozanne, \& Hill, 2016; Mick, Pettigrew, Pechmann, \& Ozanne, 2012). A TCR abraça a promessa de oferecer benefícios sociais ao não acadêmicos, ao mesmo tempo que contribui com o desenvolvimento conceitual para os acadêmicos, de modo que cada foco se beneficie de forma interativa com o outro. É um movimento de pesquisadores que desejam ser relevantes em questões como pobreza, sustentabilidade, bem-estar de alimentos, conflitos sociais, estigmas e injustiças, para citar alguns dos problemas que precisam ser abordados, como o da sustentabilidade ambiental (Davis et al., 2016).

No Brasil, ainda não tem um corpo muito grande de pesquisas sobre esses temas. Porém, segundo de (Pinto, Batinga, Ássimos, \& Almeida, 2016), foram publicados 15 trabalhos sobre essa temática durante o período de 2008 a 2016. O primeiro, em 2008, é um trabalho teórico sobre o consumo e o meio ambiente. Outros onze trabalhos são sobre os consumidores deficientes. Os demais foram um ensaio teórico, um sobre mulheres materialistas e um sobre o turismo voluntário. Observa-se que ainda não há uma extensão nos trabalhos visando o comportamento pró-ambiental como antecedente de bem-estar, podendo este trabalho estar contribuindo para esse campo do comportamento do consumidor.

No entanto, o movimento da pesquisa do consumidor para a pesquisa transformativa do consumidor requer pensamento, planejamento e ajuste contínuos. Opera-se em um ambiente institucional que nem sempre pode ser propício a diferentes formas de autoria e novas maneiras de promover mais estudos de ativistas (Mick, Pettigrew, Pechmann, Ozanne, et al., 2012). 0 movimento está plenamente ciente de que uma parcela mínima de bens e serviços é necessária para a sobrevivência, mas também deve-se encontrar maneiras de melhorar a qualidade de vida dos consumidores que garantam oportunidades para prosperar também. 0 movimento do TCR já mudou a pesquisa acadêmica, e deve continuar a proliferar enquanto houver trabalho conjunto, em colaboração com cidadãos e não acadêmicos, para criar um futuro de bem-estar compartilhado (Davis et al., 2016).

No campo do comportamento do consumidor, há um crescente coro de pesquisadores consumidores transformativos que pedem que o marketing tenha um impacto social positivo (Mick, Pettigrew, Pechmann, Ozanne, et al., 2012). Essa integração entre as questões ambientais e o bem-estar no marketing ampliaria a visão do TCR. O Journal of Public Policy and Marketing teve edição especial, focada em pesquisas dentro do escopo do TCR, em 2011. O pensamento atual se traduz em comportamento individual e tal alteração na definição de valor depende dos consumidores que integram as considerações ambientais, como um componente central da avaliação do consumo (Polonsky, 2011). Ser transformador requer uma mudança no pensamento, que é o cerne do problema.

\section{DISCUSSÃO}

Apesar do chamado mundial ao interesse pelos comportamentos ambientais, de acordo com a Declaração do Milênio das Nações Unidas, o respeito insuficiente pela natureza é um dos maiores problemas para o desenvolvimento sustentável do nosso planeta (United Nations, 2000). E, apesar dos esforços atuais, esforços adicionais substanciais devem ser empreendidos para promover ativamente práticas de consumo ecológico em escala mundial (Martin \& Czellar, 2017).

Falta motivação para se comportar, e aprender a comportar-se, de forma ambientalmente amigável. Um exemplo disso é que seria possível reduzir $20 \%$ das emissões de carbono causadas pelas famílias nos EUA, todos os anos, se elas usassem tecnologias já disponíveis e adotassem comportamentos mais efetivos (Dietz, Gardner, Gilligan, Stern, \& Vandenbergh, 2009). A maioria das pessoas está preocupada com as mudanças climáticas, mas 
poucos estão dispostos a agir para reverter o fenômeno (Gifford, 2011). As empresas não conseguem manter metas e ações em relação a isso, e o consumidor ainda não se vê como um fator essencial para expandir o comportamento pró-ambiental. À luz desses desafios urgentes, é necessário compreender melhor os fatores que contribuem para as práticas sustentáveis de consumo (Martin \& Czellar, 2017).

Para melhorar e incentivar os comportamentos ambientais, este artigo apresenta um quadro teórico que explora que variáveis que podem aumentar o comportamento ambiental e como esse comportamento pode trazer um bem-estar social aos consumidores. Assim, seria possível estimula-los a buscar ações positivas para o meio ambiente, reduzindo o impacto ambiental e trazendo bem-estar individual e coletivo. Muitas pessoas são intrinsecamente motivadas a se envolver em ações proeminentes, porque contribuir para o bem maior faz com que elas se sintam boas em relação a si mesmas (Steg, 2016).

Cabe influenciar e mostrar, para as empresas, que é possível estimular os consumidores a se portarem de maneira mais pró-ativa em relação à tomada de decisão positiva e consciente ambientalmente. Desta forma, em primeiro lugar é preciso analisar as atitudes pró-ambientais dos consumidores, o processo do consumidor que já tem uma atitude pró-ambiental e o processo de incentivar essas atitudes positivas. Em segundo lugar, é necessário identificar como outras pessoas e como as normas sociais afetam esse comportamento pró-ambiental, tentando prever quais medidas vão auxiliar no desenvolvimento de uma cultura de ações ambientais. Assim, as empresas podem descobrir que tipo de informações incentivam mais o comportamento e qual o papel da sociedade nessa influência, levando à descoberta de insights sobre 0 consumidor. Em terceiro lugar, é essencial que a empresa crie uma vantagem competitiva sustentável se beneficiando dos comportamentos ambientais dos consumidores. É previsto que, se os consumidores tiverem uma percepção de bem-estar social positiva na sua relação com todo o meio ambiente, é provável que mantenham o seu comportamento, estimulando outros consumidores e assumindo a sua responsabilidade em relação as suas decisões de escolha de produtos/serviços que sejam considerados sustentáveis.

Como indicado por (Abrahamse \& Steg, 2013), realizamos uma pesquisa empírica, relacionando como a influência social afeta a mudança de comportamento, visando contribuir e estimular que pesquisas futuras explorem ainda mais todo o potencial das abordagens de influência social para incentivar os comportamentos pró-ambientais. Como demostrado na Figura 1 unimos algumas teorias que se relacionam com a tomada de decisão respondendo ao chamado de (Farrow et al., 2017), para produzir um novo modelo a ser testado. E como (Miller \& Prentice, 2016) recomendaram análises de bem-estar mais abrangentes de intervenções de normas sociais e intervenções comportamentais de forma mais geral, neste estudo analisamos em específico o CPA, que através da análise dos estudos citados neste trabalho, intuímos que o CPA é um preditor de bem-estar ao consumidor.

A seguir, apresenta-se as proposições de pesquisa a serem analisadas após a discussão e escolha das variáveis.

P1: As atitudes pró-ambientais de um consumidor afetam diretamente os comportamentos pró-ambientais de outros, aumentando esses comportamentos positivos.

P2: As normas sociais afetam positivamente o comportamento pró-ambiental aumentando a percepção de bem-estar social do consumidor.

P2a: As normas sociais aumentam as atitudes pró-ambientais assim aumentando os comportamentos pró-ambientais.

P3: 0 bem-estar social modera a relação entre as normas sociais e o comportamento próambiental.

P4: As atitudes pró-ambientais dos consumidores afetam a relação das normas sociais aumentando o comportamento próambiental e o bem-estar social.

P4a: As atitudes pró-ambientais moderam a relação entre as normas sociais e os comportamentos pró-ambientais.

Exploramos alguns fatores contextuais determinam o tipo de atitude (Steg \& Vlek, 2009) como citado pelos autores normas pessoais, crenças e valores, delineados na Teoria da Norma de Crença de Valor, que no caso dos nossos resultados afetam e motivam os 
consumidores ao CPA. Consideramos também a atitude, e as normas sociais como mediadores da relação como em (Lee et al., 2014) os autores não consideram essa lacuna no modelo de medição, mas indicaram para pesquisa futura. Desta forma, e com base nos estudos analisados anteriormente, foi construída a Figura 1, que ilustra o quadro conceitual. No apêndice 1 deste trabalho apresenta-se uma tabela com uma relação inicial de variáveis que foram utilizadas em alguns estudos anteriores, e podem ser utilizadas (adaptadas) para testar as relações das proposições de pesquisa.

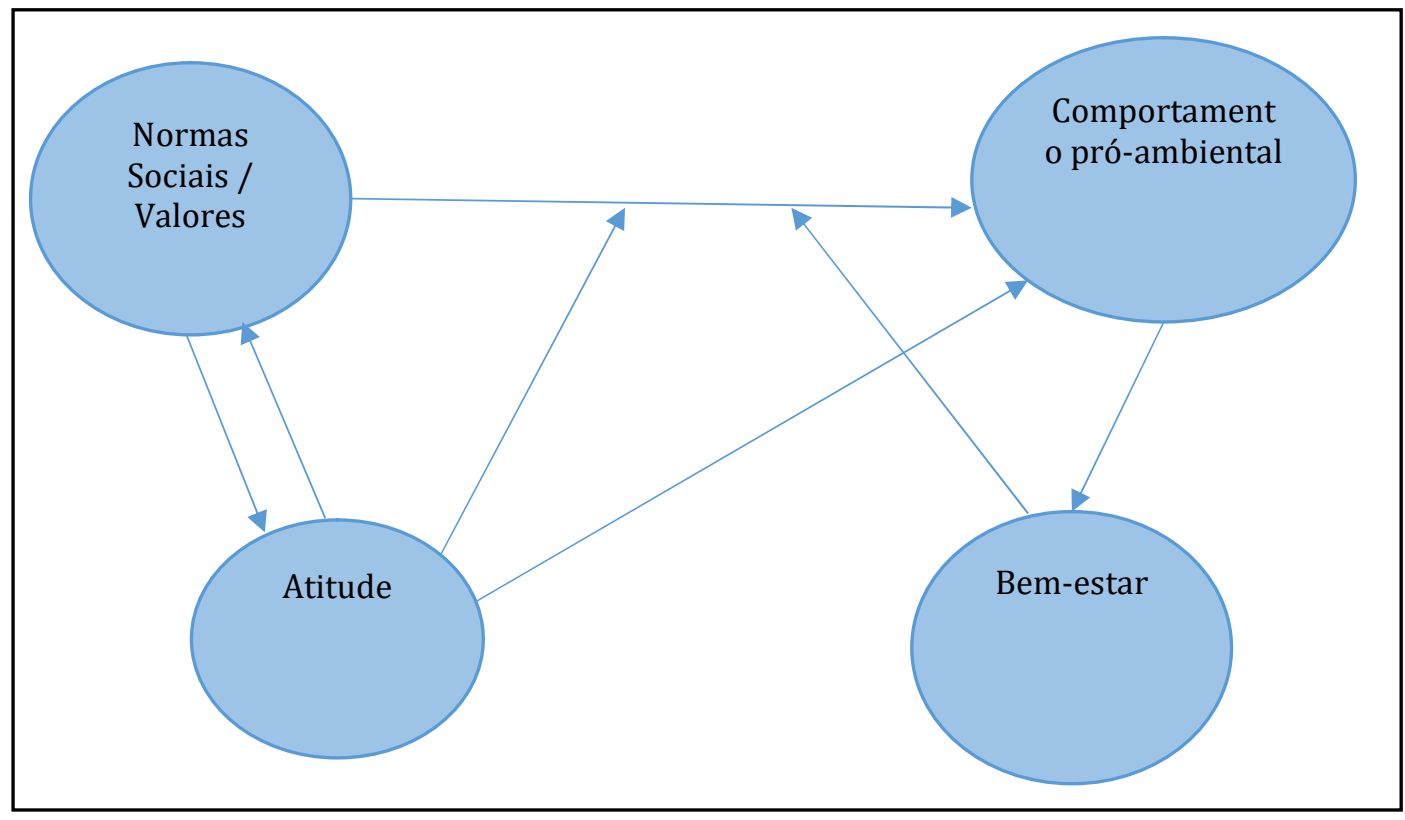

Fonte: Elaborado pelos Autores (2019).

Figura 1: Framework Teórico

Nos últimos anos, a importância da agenda ambiental para a indústria vem crescendo exponencialmente em nível internacional. As evidências empíricas sugerem que as estratégias verdes permitem a obtenção de benefícios econômicos, tanto nas operações da empresa quanto na estratégia de mercado redução de custos de produção, melhoria da posição competitiva, entrada em novos mercados - e sua posição de capital de risco aumento do poder de barganha para parceiros de capital de risco.

\section{CONCLUSÃO}

Cabe ao marketing, e aos pesquisadores de comportamento do consumidor, estimular o consumo consciente juntamente com o comportamento pró-ambiental. O Journal of Macromarketing publicou uma edição especial sobre sustentabilidade, examinando como o marketing pode ajudar a enfrentar os problemas ambientais enfrentados hoje (Kilbourne, 2010). Seguindo as mesmas questões, o Journal of Macromarketing lançou, em 2014 e 2015, duas edições especiais sobre a sustentabilidade chamadas Sustainability as Megatrend I e Sustainability as Megatrend II.

Sendo então a sustentabilidade uma questão relevante para o marketing, Polonsky (2011) traz o marketing transformativo verde, que amplia a perspectiva do consumo, incorporando questões ambientais na principal atividade de marketing. Porém, responder por mudanças nos padrões de consumo não é algo que os consumidores, as empresas, ou mesmo os governos, estão fazendo atualmente. Uma orientação cada vez mais materialista na sociedade pode complicar a transição para um futuro mais sustentável, prejudicando o apoio público a políticas ambientais mais rigorosas e reforçando práticas de consumo insustentáveis (Andersson \& Nässén, 2016). O valor depende dos consumidores, que integram as considerações ambientais como um componente central da avaliação do consumo (Polonsky, 2011). 
É preciso incentivar e mostrar ao consumidor, que medidas pró-ambientais podem ajudá-lo a alcançar o bem-estar. Esperase que esses indivíduos, que se preocupam o suficiente com questões societárias e acreditam no poder de suas ações para mudar o comportamento de outros, atuem sobre essa crença (Bret Leary, Vann, \& Mittelstaedt, 2017) e atinjam mais pessoas para a mudança para o comportamento pró-ambiental. Este trabalho se propôs a levantar a questão do comportamento pró-ambiental para estimular e promover o debate, e as ações das empresas, a fim de poder conseguir medir como se promove esses comportamentos e se é possível através do sentimento de bem-estar social trazer uma maior consciência comportamental dos consumidores.

A sustentabilidade ganhou importância significativa nos últimos anos. Em particular, os gestores são confrontados com pedidos de comportamento sustentável, considerando seu papel especial como o canal que tem maior contato com o consumidor final (Wiese, Zielke, \& Toporowski, 2015). A sustentabilidade ambiental e social tem relevância e uma presença crescente dentro do campo da gestão (Ashby, Leat, \& Hudson-Smith, 2012).

\section{Limitações e pesquisas futuras}

Embora algumas empresas já se dediquem a produzirem produtos e serviços de maneira mais sustentável, ainda é difícil fazer com que a mudança de comportamento seja realmente efetiva. Então, é necessário o envolvimento de profissionais de marketing para, efetivamente, poder mudar a estrutura e as práticas empresariais sustentáveis. 0 marketing de produtos vem usando com sucesso estratégias persuasivas, como propaganda audiovisual e campanhas de promoção, para envolver os consumidores e influenciar suas escolhas e comportamentos (Mooij, 2010). A utilização da teoria do comportamento planejado foi efetivamente usada para conceituar e medir intenções comportamentais no consumo de produtos orgânicos, bem como o consumo sustentável (Johe \& Bhullar, 2016).

Abrahamse e Steg (2013) descrevem algumas questões interessantes a serem abordadas: (1) é necessária uma pesquisa empírica mais sistemática que ligue os mecanismos de influência social à mudança de comportamento; (2) são necessários mais estudos de campo que incluem medidas de variáveis potenciais do moderador para permitir o exame das condições em que as abordagens de influência social são mais efetivas nas configurações aplicadas; e (3) é preciso analisar em quais dimensões o grupo de comparação deve ser semelhante ao grupoalvo.

Muitos problemas ambientais estão enraizados no comportamento humano e, portanto, podem ser reduzidos quando as pessoas se envolvem em ações pró-ambientais de forma mais consistente (Steg, 2016). As estratégias para encorajar ações proeminentes podem visar o fortalecimento dos valores biosféricos ou a mudança do contexto de escolha. Os valores biosféricos são mais prováveis de serem apoiados e ativados no contexto relevante onde as decisões são tomadas, capacitando as pessoas a atuarem de acordo com os valores que considerem importantes.

Embora seja claro que as normas sociais são um determinante confiável do comportamento pró-ambiental, mais estudos devem ser feitos sobre como as normas podem ser melhor alavancadas (Farrow et al., 2017). Os autores também indicam várias lacunas na teoria que podem ser analisadas: (1) ligar, mais intimamente, a pesquisa acadêmica a cenários do mundo real; (2) explorar em maior profundidade o papel dos erros de engano e julgamento na aplicação e conformidade da norma social; (3) comparar a interação das intervenções da norma social com outros tipos de intervenções; e (4) comunicar normas sociais através da combinação de normas prescritivas versus proscritivas, e descritivas versus injuntivas. A evidência apresentada (ver Figura 1) demonstra que as crenças sobre o comportamento e as atitudes dos outros são um determinante confiável do comportamento individual em relação ao meio ambiente, e que os esforços para entender melhor esse fenômeno devem ser um elemento importante na busca contínua de um ambiente ambiental efetivo.

Ainda não houve uma revisão sistemática da literatura no Brasil unindo essas variáveis, o bem-estar ainda não foi analisado como um possível resultado para os consumidores que 
possuem um comportamento pró-ambiental. Estudos futuros podem colocar em prática o Framework desenvolvido neste estudo, e analisar as relações para confirmar nossas proposições de pesquisa, e ainda examinar mais variáveis (por exemplo, orientação egoísta, compaixão). Estudos futuros podem querer medir o comportamento real (Lee et al., 2014), através de experimentos ou quaseexperimentos. Reforçado por (Wan et al., 2017) estudos futuros se beneficiariam com a inclusão de comportamentos medidos objetivos observando e registrando comportamentos reais de reciclagem, as descobertas podem ser usadas para abordar a limitação dos comportamentos auto relatos.

Buscamos entender como as normas sociais atuais se desempenham e ajudam nas mudanças de estilos de vida dos consumidores (Steentjes et al., 2017), e como as mensagens de manipulação de normas, o feedback socialmente comparativo e os ambientes sociais normativos afetam as ações individuais (Keizer, Lindenberg, \& Steg, 2008; Schultz, Nolan, Cialdini, Goldstein, \& Griskevicius, 2007; Vossen, Ham, \& Midden, 2009). Pois através de atitudes e normas socias podemos prever e incentivar certos comportamentos evidenciando o resultado final, bem-estar ao consumidor.

A luta humana fundamental para o bemestar e a felicidade subjetiva, na vida cotidiana, é uma força motriz mais provável para a mudança (Brown \& Vergragt, 2016), já que a transição para uma economia que está em harmonia com os limites ecológicos é uma das maiores questões contemporâneas. 0 crescimento econômico ilimitado, com base no consumo de materiais em massa, é insustentável e uma receita para o desastre. Promover o trabalho sério, trazer essas questões em forma de um debate e de pesquisas, pode trazer benefícios reais e aplicados à sociedade, assim como pensar que é possível promover uma mudança ambiental.

\section{REFERÊNCIAS}

Abrahamse, W., \& Steg, L. (2013). Social influence approaches to encourage resource conservation: A meta-analysis. Global Environmental Change, 23(6), 1773-1785.
Ajzen, I. (1991a). The theory of planned behavior. Organizational Behavior and Human Decision Processes, 50(2), 179-211.

Ajzen, I. (1991b). The theory of planned behavior. Organizational Behavior and Human Decision Processes, 50(2), 179-211.

Anderson, L., Ostrom, A. L., Corus, C., Fisk, R. P., Gallan, A. S., Giraldo, M., ... Williams, J. D. (2013). Transformative service research: An agenda for the future. Journal of Business Research, 66(8), 1203-1210.

Andersson, D., \& Nässén, J. (2016). Should environmentalists be concerned about materialism? An analysis of attitudes, behaviours and greenhouse gas emissions. Journal of Environmental Psychology, 48, 1-11.

Ashby, A., Leat, M., \& Hudson-Smith, M. (2012). Making connections: a review of supply chain management and sustainability literature. Supply Chain Management: An International Journal, 17(5), 497-516.

Barbarossa, C., De Pelsmacker, P., \& Moons, I. (2017). Personal Values, Green Self-identity and Electric Car Adoption. Ecological Economics, 140, 190-200.

Binder, M., \& Blankenberg, A.-K. (2017). Green lifestyles and subjective well-being: More about self-image than actual behavior? Journal of Economic Behavior \& Organization, 137, 304-323.

Bissing-Olson, M. J., Fielding, K. S., \& Iyer, A. (2016). Experiences of pride, not guilt, predict proenvironmental behavior when proenvironmental descriptive norms are more positive. Journal of Environmental Psychology, 45, 145-153.

Bret Leary, R., Vann, R. J., \& Mittelstaedt, J. D. (2017). Leading the way: Motivating environmental action through perceived marketplace influence. Journal of Business Research, 79(May), 79-89.

Broek, K. Van Den, Willem, J., \& Steg, L. (2017). Individual differences in values determine the relative persuasiveness of biospheric, economic and combined appeals. Journal of Environmental Psychology, 53, 145-156.

Brown, H. S., \& Vergragt, P. J. (2016). From consumerism to wellbeing: toward a cultural transition? Journal of Cleaner Production, 132, 308-317.

Bublitz, M. G., Peracchio, L. A., Andreasen, A. R., Kees, J., Kidwell, B., Miller, E. G., Vallen, B. (2013). Promoting positive change: Advancing the food well-being paradigm. Journal of Business Research, 66(8), 1211-1218.

Carfora, V., Caso, D., Sparks, P., \& Conner, M. (2017). Moderating effects of pro-environmental selfidentity on pro-environmental intentions and 
behaviour: A multi-behaviour study. Journal of Environmental Psychology, 53, 92-99.

Cialdini, R. B. (2003). Crafting Normative Messages to Protect the Environment. Current Directions in Psychological Science, 12(4), 105-109.

Cialdini, R. B., \& Goldstein, N. J. (2004). Social Influence: Compliance and Conformity. Annual Review of Psychology, 55(1), 591-621.

Cialdini, R. B., Kallgren, C. A., \& Reno, R. R. (1991). A Focus Theory of Normative Conduct: A Theoretical Refinement and Reevaluation of the Role of Norms in Human Behavior (pp. 201234).

Cialdini, R. B., \& Trost, M. R. (1998). Social influence: Social norms, conformity and compliance. New York, NY: McGraw-Hill.

Coelho, F., Pereira, M. C., Cruz, L., Simões, P., \& Barata, E. (2017). Affect and the adoption of pro-environmental behaviour: A structural model. Journal of Environmental Psychology, 54, 127-138.

Davis, B., Ozanne, J. L., \& Hill, R. P. (2016). The Transformative Consumer Research Movement. Journal of Public Policy \& Marketing, 35(2), 159-169.

Davis, B., \& Pechmann, C. (2013). Introduction to the Special Issue on transformative consumer research: Developing theory to mobilize efforts that improve consumer and societal well-being. Journal of Business Research, 66(8), 1168-1170.

Diener, E., Lucas, R., Schimmack, U., \& Helliwell, J. (2009). Well-Being for Public Policy. Oxford University Press.

Dietz, T., Gardner, G. T., Gilligan, J., Stern, P. C., \& Vandenbergh, M. P. (2009). Household actions can provide a behavioral wedge to rapidly reduce US carbon emissions. Proceedings of the National Academy of Sciences of the United States of America, 106(44), 18452-18456.

Dunlap, R. E., Van Liere, K. D., Mertig, A. G., \& Jones, R. E. (2000). New Trends in Measuring Environmental Attitudes: Measuring Endorsement of the New Ecological Paradigm: A Revised NEP Scale. Journal of Social Issues, 56(3), 425-442.

Ertz, M., Karakas, F., \& Sarigöllü, E. (2016). Exploring pro-environmental behaviors of consumers: An analysis of contextual factors, attitude, and behaviors. Journal of Business Research, 69(10), 3971-3980.

Farrow, K., Grolleau, G., \& Ibanez, L. (2017). Social Norms and Pro-environmental Behavior: A Review of the Evidence. Ecological Economics, 140, 1-13.

Gifford, R. (2011). The dragons of inaction: psychological barriers that limit climate change mitigation and adaptation. American Psychologist, 66(4), 290-302.

Huang, H. (2016). Media use, environmental beliefs, self-efficacy, and pro-environmental behavior. Journal of Business Research, 69(6), 22062212.

Hynes, N., \& Wilson, J. (2016). I do it, but don't tell anyone! Personal values, personal and social norms: Can social media play a role in changing pro-environmental behaviours? Technological Forecasting and Social Change, 111, 349-359.

IPCC. (2014). INTERGOVERNMENTAL PANEL ON CLIMATE CHANGE. Retrieved June 17, 2018, from https://www.ipcc.ch/pdf/assessmentreport/ar5/wg2/ar5_wgII_spm_en.pdf

Johe, M. H., \& Bhullar, N. (2016). To buy or not to buy: The roles of self-identity, attitudes, perceived behavioral control and norms in organic consumerism. Ecological Economics, 128, 99-105.

Kaida, N., \& Kaida, K. (2016). Pro-environmental behavior correlates with present and future subjective well-being. Environment, Development and Sustainability, 18(1), 111127.

Kaiser, F. G. (2006). A moral extension of the theory of planned behavior: Norms and anticipated feelings of regret in conservationism. Personality and Individual Differences, 41(1), 71-81.

Keizer, K., Lindenberg, S., \& Steg, L. (2008). The spreading of disorder. Science (New York, N.Y.), 322(5908), 1681-1685.

Keizer, K., \& Schultz, P. W. (2018). Social Norms and Pro-Environmental Behaviour. In L. Steg \& J. I. De Groot (Eds.), Environmental Psychology (pp. 179-188). Chichester, UK: John Wiley \& Sons,

Kilbourne, W. E. (2010). Facing the Challenge of Sustainability in a Changing World: An Introduction to the Special Issue. Journal of Macromarketing, 30(2), 109-111.

Kitayama, S., Markus, H. R., \& Kurokawa, M. (2000). Culture, Emotion, and Well-being: Good Feelings in Japan and the United States. Cognition \& Emotion, 14(1), 93-124.

Kjell, O. N. E. (2011). Sustainable well-being: A potential synergy between sustainability and well-being research. Review of General Psychology, 15(3), 255-266.

Kumar, B., Manrai, A. K., \& Manrai, L. A. (2017). Purchasing behaviour for environmentally sustainable products: A conceptual framework and empirical study. Journal of Retailing and Consumer Services, 34, 1-9.

Lee, Y., Kim, S., Kim, M., \& Choi, J. (2014). Antecedents and interrelationships of three 
types of pro-environmental behavior. Journal of Business Research, 67(10), 2097-2105.

LUCAS, R. (2016). Subjective well-being in psychology. In The Oxford Handbook of WellBeing and Public Policy (pp. 403-423).

Martin, C., \& Czellar, S. (2017). Where do biospheric values come from? A connectedness to nature perspective. Journal of Environmental Psychology, 52, 56-68.

Mick, D. G., Pettigrew, S., Pechmann, C. C., \& Ozanne, J. L. (2012). Transformative consumer research for personal and collective well-being (Taylor \& F). New York, NY: Routledge.

Mick, D. G., Pettigrew, S., Pechmann, C., Ozanne, J. L., Pettigrew, S., Pechmann, C., \& Ozanne, J. L. (2012). Origins, Qualities, and Envisionments of Transformative Consumer Research, 31-52.

Miller, D. T., \& Prentice, D. A. (2016). Changing Norms to Change Behavior. Annual Review of Psychology, 67(1), 339-361.

Mooij, M. K. de. (2010). Consumer behavior and culture: consequences for global marketing and advertising. Sage Publications.

Pinto, M. de R., Batinga, G. L., Ássimos, B. M., \& Almeida, G. T. (2016). Transformative Consumer Research (TCR): Reflexões, Diretrizes e uma Análise do Campo no Brasil. Revista Interdisciplinar de Marketing, 6(2), 5466.

Polonsky, M. J. (2011). Transformative green marketing: Impediments and opportunities. Journal of Business Research, 64(12), 13111319.

Robinson, R., \& Smith, C. (2002). Psychosocial and Demographic Variables Associated with Consumer Intention to Purchase Sustainably Produced Foods as Defined by the Midwest Food Alliance. Journal of Nutrition Education and Behavior, 34(6), 316-325.

Rucker, D. D., Tormala, Z. L., Petty, R. E., \& Briñol, P. (2014). Consumer conviction and commitment: An appraisal-based framework for attitude certainty. Journal of Consumer Psychology, 24(1), 119-136.

Ruepert, A., Keizer, K., Steg, L., Maricchiolo, F., Carrus, G., Dumitru, A., ... Moza, D. (2016). Environmental considerations in the organizational context: A pathway to proenvironmental behaviour at work. Energy Research and Social Science, 17, 59-70.

Schmitt, M. T., Aknin, L. B., Axsen, J., \& Shwom, R. L. (2018). Unpacking the Relationships Between Pro-environmental Behavior, Life Satisfaction, and Perceived Ecological Threat. Ecological Economics, 143, 130-140.

Schröder, T., \& Wolf, I. (2017). Modeling multi-level mechanisms of environmental attitudes and behaviours: The example of carsharing in
Berlin. Journal of Environmental Psychology, 52, 136-148.

Schultz, P. W., Nolan, J. M., Cialdini, R. B., Goldstein, N. J., \& Griskevicius, V. (2007). The Constructive, Destructive, and Reconstructive Power of Social Norms. Psychological Science, 18(5), 429-434.

Schultz, P. W., \& Zelezny, L. C. (1998). Values and Proenvironmental Behavior. Journal of CrossCultural Psychology, 29(4), 540-558.

Schwartz, S. H. (1992). Universals in the content and structure of values: Theoretical advances and empirical tests in 20 countries. Advances in Experimental Social Psychology, 25(C), 1-65.

Schwartz, S. H., Cieciuch, J., Vecchione, M., Davidov, E., Fischer, R., Beierlein, C., ... Schwartz, H. (2012). Refining the Theory of Basic Individual Values. Journal of Personality and Social Psychology, 103(4), 663-688.

Searle, B. A. (2008). Well-being : in search of a good life? Bristol: The Plice Press.

Sheng, X., Siguaw, J. A., \& Simpson, P. M. (2016). Servicescape attributes and consumer wellbeing. Journal of Services Marketing, 30(7), 676-685.

Steentjes, K., Kurz, T., Barreto, M., \& Morton, T. A. (2017). The norms associated with climate change: Understanding social norms through acts of interpersonal activism. Global Environmental Change, 43, 116-125.

Steg, L. (2016). Values, norms, and intrinsic motivation to act proenvironmentally. Annual Review of Environment and Resources, 41(1), 277-292.

Steg, L., \& Vlek, C. (2009). Encouraging proenvironmental behaviour: An integrative review and research agenda. Journal of Environmental Psychology, 29(3), 309-317.

Stern, P. C. (2000). New Environmental Theories: Toward a Coherent Theory of Environmentally Significant Behavior. Journal of Social Issues, 56(3), 407-424.

Vossen, S., Ham, J., \& Midden, C. (2009). Social influence of a persuasive agent. In Proceedings of the 4th International Conference on Persuasive Technology - Persuasive '09 (p. 1). New York, New York, USA: ACM Press.

Wan, C., Shen, G. Q., \& Choi, S. (2017). Experiential and instrumental attitudes: Interaction effect of attitude and subjective norm on recycling intention. Journal of Environmental Psychology, 50, 69-79.

Welsch, H., \& Kühling, J. (2018). How Green Self Image is Related to Subjective Well-Being: Pro-Environmental Values as a Social Norm. Ecological Economics, 149, 105-119.

Wiese, A., Zielke, S., \& Toporowski, W. (2015). Sustainability in retailing - research streams 
and emerging trends. International Journal of Retail \& Distribution Management, 43(4/5), IJRDM-02-2015-0024.

Yang, D., Kao, W. T. M., Huang, N., Wang, R., Zhang, X., \& Zhou, W. (2014). Process-based environmental communication and conflict mitigation during sudden pollution accidents. Journal of Cleaner Production, 66.

\title{
Examining the Domain of Pro-Environmental Behavior to Promote Individual and Collective Well- being
}

\begin{abstract}
The analysis of consumer behavior is at the center of an assessment (evolution) of the environmental and sustainable sustainability of society. Pro-environmental behavior can help minimize man's impact on the environment. This research delineates a conceptual framework that is based on social norms, values and pro-environmental attitudes to promote a pro-environmental behavior with contributions to the transformative consumer research and personal and collective well-being. In addition, to better understand how one can stimulate this behavior in various marketing activities, it is discussed how companies and society can benefit from these pro-environmental behaviors, allowing organizations to better exploit their advantages.
\end{abstract}

Keywords: Well-being, Pro-environmental behavior, Pro-environmental attitudes, Transformative Consumer Research.

\section{Apêndice}

Tabela 1

Relação de variáveis utilizadas em estudos sobre comportamento ambiental

\begin{tabular}{|c|c|c|c|c|c|}
\hline Autor(es) & $\begin{array}{c}\text { Variáveis } \\
\text { dependentes }\end{array}$ & $\begin{array}{c}\text { Variáveis } \\
\text { independes }\end{array}$ & Mediador & $\begin{array}{l}\text { Tipo de } \\
\text { artigo e } \\
\text { método }\end{array}$ & $\begin{array}{c}\text { Fornece } \\
\text { itens da } \\
\text { Escala } \\
\end{array}$ \\
\hline $\begin{array}{l}\text { DUNLAP, et al. } \\
(2000)\end{array}$ & $\begin{array}{l}\text { Visão de mundo } \\
\text { ecológica }\end{array}$ & $\begin{array}{c}\text { Limites para o } \\
\text { crescimento; anti- } \\
\text { antropocentrismo; } \\
\text { fragilidade do } \\
\text { equilíbrio da } \\
\text { natureza; rejeição do } \\
\text { exemptionalism; } \\
\text { possibilidade de uma } \\
\text { ecocrisis; saldo }\end{array}$ & - & $\begin{array}{c}\text { Empírico, } \\
\text { Quantitativo; } \\
\text { Desenvolvime } \\
\text { nto de escala }\end{array}$ & Sim \\
\hline $\begin{array}{l}\text { CIALDINI } \\
(2003)\end{array}$ & $\begin{array}{l}\text { Intenção de } \\
\text { reciclar }\end{array}$ & $\begin{array}{l}\text { Normas injuntivas, } \\
\text { normas descritivas }\end{array}$ & - & $\begin{array}{c}\text { Empírico, } \\
\text { quantitativo; } \\
\text { Experimento }\end{array}$ & $\begin{array}{c}\text { Métodos } \\
\text { de coleta } \\
\text { oferecidos }\end{array}$ \\
\hline $\begin{array}{l}\text { LEE, Yong-ki et } \\
\text { al. (2014) }\end{array}$ & $\begin{array}{c}3 \text { tipos de } \\
\text { comportamento } \\
\text { ambiental: Bom } \\
\text { comportamento de } \\
\text { cidadania; } \\
\text { Comportamento } \\
\text { de compra verde; } \\
\text { Comportamento }\end{array}$ & $\begin{array}{l}\text { Orientação ao valor } \\
\text { altruísta; eficácia do } \\
\text { consumidor } \\
\text { percebida (PCE); } \\
\text { preocupação } \\
\text { ambiental. }\end{array}$ & - & $\begin{array}{c}\text { Empírico, } \\
\text { quantitativo; } \\
\text { Survey }\end{array}$ & Sim \\
\hline
\end{tabular}




\begin{tabular}{|c|c|c|c|c|c|}
\hline & $\begin{array}{l}\text { do ativista } \\
\text { ambiental }\end{array}$ & & & & \\
\hline $\begin{array}{l}\text { BISSING- } \\
\text { OLSON; } \\
\text { FIELDING; } \\
\text { IYER (2016) }\end{array}$ & $\begin{array}{l}\text { Comportamento } \\
\text { pró-ambiental }\end{array}$ & $\begin{array}{l}\text { Normas sociais; } \\
\text { Normas descritivas; } \\
\text { Orgulho; Culpa }\end{array}$ & $\begin{array}{c}\text { Normas } \\
\text { descritivas }\end{array}$ & $\begin{array}{c}\text { Empírico, } \\
\text { quantitativo; } \\
\text { Experimento }\end{array}$ & $\begin{array}{l}\text { Todos os } \\
\text { itens de } \\
\text { análise } \\
\text { oferecidos }\end{array}$ \\
\hline $\begin{array}{l}\text { ERTZ; } \\
\text { KARAKAS; } \\
\text { SARIGÖLLÜ } \\
(2016)\end{array}$ & $\begin{array}{l}\text { Comportamento } \\
\text { de esfera privada; } \\
\text { Comportamento } \\
\text { de esfera pública }\end{array}$ & $\begin{array}{c}\text { Fatores } \\
\text { contextuais: } \\
\text { ocupação } \\
\text { perceptível; riqueza } \\
\text { percebida; poder } \\
\text { percebido; } \\
\text { Importância }\end{array}$ & $\begin{array}{l}\text { Atitude: } \\
\text { Importância; } \\
\text { duração; } \\
\text { custo }\end{array}$ & $\begin{array}{l}\text { Empírico, } \\
\text { quantitativo; } \\
\text { Survey }\end{array}$ & Não \\
\hline $\begin{array}{l}\text { CONNER et al. } \\
(2016)\end{array}$ & $\begin{array}{c}\text { Intenção- } \\
\text { comportamento }\end{array}$ & $\begin{array}{c}\text { objetivo e } \\
\text { a estabilidade da } \\
\text { intenção; intenção no } \\
\text { tempo; intenção, } \\
\text { prioridade de } \\
\text { objetivo, conflito de } \\
\text { objetivos }\end{array}$ & - & $\begin{array}{c}\text { Empírico, } \\
\text { quantitativo; } \\
\text { Experimento }\end{array}$ & Sim \\
\hline HUANG (2016) & $\begin{array}{l}\text { Comportamento } \\
\text { pró-ambiental; } \\
\text { Comportamento } \\
\text { de acomodação; } \\
\text { Comportamento } \\
\text { promocional; } \\
\text { Comportamento } \\
\text { proativo }\end{array}$ & $\begin{array}{l}\text { O uso de mídia de } \\
\text { aquecimento global; } \\
\text { Crenças ambientais; } \\
\text { Auto eficácia } \\
\text { ambiental }\end{array}$ & - & $\begin{array}{l}\text { Empírico, } \\
\text { quantitativo, } \\
\text { Survey }\end{array}$ & Sim \\
\hline $\begin{array}{l}\text { WAN; SHEN; } \\
\text { CHOI (2017) }\end{array}$ & $\begin{array}{l}\text { Intenção de } \\
\text { reciclar }\end{array}$ & $\begin{array}{c}\text { Atitude; Norma } \\
\text { subjetiva; Controle } \\
\text { comportamental } \\
\text { percebido; } \\
\text { Consciência de } \\
\text { consequências; } \\
\text { Norma moral }\end{array}$ & - & $\begin{array}{l}\text { Empírico, } \\
\text { quantitativo; } \\
\text { Survey }\end{array}$ & Não \\
\hline $\begin{array}{l}\text { CARFORA, V. et } \\
\text { al. (2017) }\end{array}$ & $\begin{array}{c}\text { Comportamento } \\
\text { ambiental }\end{array}$ & $\begin{array}{c}\text { Perceived } \\
\text { Behavioural Control; } \\
\text { Auto-identidade; } \\
\text { Intenção; Atitude; } \\
\text { Norma subjetiva }\end{array}$ & - & $\begin{array}{c}\text { Empírico, } \\
\text { quantitativo; } \\
\text { Survey }\end{array}$ & Sim \\
\hline $\begin{array}{l}\text { LEARY; VANN; } \\
\text { MITTELSTAED } \\
\text { T (2017) }\end{array}$ & $\begin{array}{c}\text { Comportamento } \\
\text { ambiental }\end{array}$ & $\begin{array}{l}\text { Ambientalismo } \\
\text { social; Percepção da } \\
\text { influência do } \\
\text { mercado PMI: (PMI } \\
\text { Consumer e PMI } \\
\text { Organization); } \\
\text { Comportamento eco- } \\
\text { consciente do } \\
\text { consumidor; } \\
\text { Ceticismo do } \\
\text { consumidor; } \\
\text { Ambientalismo } \\
\text { social; Cidadania } \\
\text { ambiental }\end{array}$ & $\begin{array}{l}\text { Ceticismo do } \\
\text { consumidor }\end{array}$ & $\begin{array}{c}\text { Empírico, } \\
\text { quantitativo; } \\
\text { Survey e } \\
\text { Validação } \\
\text { escala }\end{array}$ & Sim \\
\hline
\end{tabular}

Fonte: Elaborado pelos Autores (2019). 